\title{
el Ambiente Compartido como Propulsor de la INTERNACIONALIZACIÓN: ANÁLISIS A PARTIR DE LA GESTIÓN DEL CONOCIMIENTO Y DE LA INNOVACIÓN
} .

\author{
O Ambiente Compartilhado como Propulsor de \\ Internacionalização: análise da gestão do conhecimento e da \\ inovação
}

Rafael Pereira Ocampo Moré

Mestre em Administração. Universidade do Vale do Itajaí (Univali), Florianópolis, SC, Brasil. E-mail: rafamore@gmail.com

\section{Cláudio Reis Gonçalo}

Doutor. Universidade do Vale do Itajaí. Florianópolis, SC, Brasil. E-mail: claudio.goncalo@univali.br

\section{Lourdes Cános Darós}

Doctora en Economía. Universitat Politècnica de València, Espanha. E-mail: loucada@omp.upv.es

\section{Cristina Santandreu Mascarell}

Licenciada en Administración y Dirección de empresas Universitat Politècnica de València. Campus de Gandia. Valência, Espanha. E-mail: crisanma@omp.upv.es

\section{Resumen}

Este artículo analiza la internacionalización e innovación de empresas en red o que se encuentren en un entorno de investigación geográficamente cercanas para la transferencia de conocimientos. En este contexto, se investigan los modelos de internacionalización que pueden llevarse a cabo en entornos complejos, con el fin de buscar la gestión eficiente de la innovación de productos o servicios para acceder mercados globales. Así, los estudios presentados señalan relación entre las teorías de la internacionalización, gestión del conocimiento e innovación, y gestores puden hacer uso de esta relación para evaluar mejor su organización y proponer soluciones para el mercado.

Palabras clave: Innovación. Innovatividad. Internacionalización. Gestión del Conocimiento.

\section{Resumo}

Este artigo aborda a internacionalização e a inovação de empresas de rede ou que estão em um ambiente de pesquisa geograficamente próximos à transferência de conhecimentos. Nesse contexto, os modelos de internacionalização que podem ser implementados em ambientes complexos, a fim de buscar uma gestão eficaz da inovação de produtos ou serviços para acessar os mercados globais, são investigados. Assim, os estudos apresentados indicam relação entre as teorias de internacionalização, gestão do conhecimento e da inovação, e os gerentes podem usar esse relacionamento para avaliar melhor a sua organização e propor soluções para o mercado.

Palavras-chave: Inovação. Capacidade de inovação. Internacionalização. Gestão do Conhecimento. 


\section{INTROducCión}

Este trabajo analiza teóricamente el desarrollo de la internacionalización en empresas a través de la innovación y de la transferencia de conocimiento, especialmente aquellas que están interconectadas en red o geográficamente cercanas en un entorno de investigación. Este entorno, en el caso específico de su ambiente físico, se ejemplifica como un districto de innovación, parques tecnológicos o parques científicos, espacios donde las empresas pueden compartir sus conocimientos en red para optimizar sus procesos de innovación y obtener los mejores resultados. (PIQUÉ, 2014)

Un distritode innovación, por ejemplo, tiene todos los ementos necesarios para la promoción de la salude y del trabajo, baseado en la concentración de universidades y empresas en territorios con sofisticada infraestructura, coexistente con áreas residenciales y espacios públicos. (MOLAS; PARELLADA, 2014)

La red también comprende una concentración de empresas y conocimiento, que pertenece a un grupo que puede proporcionar acceso a proveedores especializados, servicios y recursos humanos. Además, la información específica tiende a circular en una red que beneficia a las empresas que permite reaccionar y adaptarse rápidamente a los cambios del mercado, como también adoptar la innovación. Por supuesto, además de estos factores informales, hay otros formales como las políticas de apoyo que pueden satisfacer las necesidades del grupo de empresarios. (CHEVALLARD; DUCH, 2014)

En la red es posible identificar grupos de instituciones formales e informales actuando. Instituciones formales respetan las diferencias entre los países debido a las regulaciones de empleo, los sistemas de propiedad intelectual y los regímenes de apropiabilidad, sistemas de negocio o reglas de operación, el funcionamiento de los mercados financeiros, los niveles de estabilidad fiscal y económico. Sin embargo, instituciones informales son sistemas con informacioes implícitas en valores, creencias y en la compreensión colectiva de la sociedad, una vez que no están codificados en normas o documentos. (FILIOU; GOLESORKHI, 2014)

En la gestión del conocimiento es importante destacar que la capacidad de absorción en una estructura informal de organizaciones permite a ellas estabelecer, por primero, un lenguaje común que facilita su colaboración y, en segundo, construir rutinas inter-organiza- cionales para la gestión eficaz de su interacción. Estas rutinas incluyen patrones de acción para la solución conjunta de problemas, el cumplimiento contractual, el intercambio de la propiedad intelectual y mejoran los resultados empresariales de las alianzas. (LANE; KOKA; PATHAK, 2006; MOLERO; GARCIA, 2008; PADILLA-PÉREZ; GAUDIN, 2014)

Además, como las diferencias de comportamientos en las estructuras instituciones informales aumentan en alianzas internacionales, los gestores deben estar cada vez más atentos, conscientes y perceptivas del cómo estas diferencias pueden crear obstáculos en el intercambio de conocimientos. Sin embargo, aumentar la percepción de las diferencias podría facilitar la agilidad e interés del gestor en invertir tiempo para la realización de alianzas necesarias para gestionar de forma correcta las diferencias. (PADILLA-PÉREZ; GAUDIN, 2014)

En entornos globales de empresas, la red puede ayudar a la organización en el desarrollo de nuevos modelos de gestión donde hay empresas integradas. La participación conjunta de las empresas permite compartir los conocimientos con el objetivo de reducir costes, y ofrecer mejores productos y servicios a sus clientes a través de la internacionalización. (COLEMAN, 1990; NOHRIA; ECCLES, 1992; AMATO, 2000; MOTTA; VASCONCELOS, 2004; YANGA; MOTOHASHIB; CHENC, 2009; AUTIO, 2014)

El concepto moderno de la red se refleja en los modelos de gestión que utilizan técnicas innovadoras de intercambio de conocimientos para mejorar el rendimiento del negocio, y son un gran reto para las empresas en red, como por ejemplo, las empresas que estan en los parques científicos. Esas acciones pueden optimizar el rendimiento de las empresas del parque, reducir costes y mejorar la gestión del sistema. (NOHRIA; ECCLES, 1992; MOTTA; AMATO, 2000; VASCONCELOS, 2004; MONTORO-SÁNCHEZ; MORA-VALENTÍN; URBINA-CRIADO, 2012; FILIOU; GOLESORKHI, 2014)

En sistemas conectados hay factores determinantes en la formación de una red interinstitucional: la diferenciación, la interdependencia y la flexibilidad. En un entorno innovador e integrado la diferenciación promueve la innovación sin aumentar los costes; la interdependencia es un factor que crea la motivación para formación de la red; y la flexibilidad es un generador de ventajas competitivas porque concede gran poder de 
adaptación de las empresas, lo que les permite cambiar en función de las contingencias del entorno empresarial. (AMATO, 2000; BERRY; GUILLEN; ZHOU, 2010; VASUDEVA; SPENCER; TEEGEN, 2013)

En cuanto a la perspectiva de la internacionalización de empresas, Álvarez y Molero (2005) investigaron el proceso de internacionalización en empresas españolas y llegaron a la conclusión de que el potencial de internacionalización aumenta cuando están en un entorno de red que permite mejorar su desempeño, por ejemplo con incentivos fiscales del gobierno $e$ intercambio de conocimientos con otras empresas. En este sentido, Rothwell (1994) analiza que la innovación se produce por el aumento de las alianzas estratégicas; con la colaboración de I+D; con la mejor gestión de la cadena de suministro; y en especial con el crecimiento de las redes entre pequeñas y medianas empresas (PYME) o entre PYME y grandes empresas.

Teniendo en cuenta lo expuesto, en este ensayo teórico presentamos como pregunta de investigación: ¿Cómo la teoría de la gestión del conocimiento y de la innovación contribuyen al desarrollo de la internacionalización de las empresas que han compartido un mismo espacio físico?

En el pasado, los negocios globales se han expandido desde países desarrollados a países en desarrollo y en la actualidad mundial la concurrencia hace que cualquier empresa pueda competir en lugares distintos y con cualquier negocio (SIRKIN; HEMERLING; BHATTACHARYA, 2008). Esto demuestra la importancia de que las empresas deben estar preparadas para competir a nivel mundial, y el ambiente debe ayudar a las empresas a desarrollar la internacionalización con acciones estratégicas e innovadoras que permitan reducir costes y buscar nuevos mercados.

Analizar empresas en parques científicos o distritos de innovación facilita la comprensión de cómo espacios innovadores e integrados pueden ayudar en el desarrollo de nuevos negocios del parque, atraer nuevas empresas, estimular la búsqueda de nuevos mercados y si el gasto de mantenimiento de este entorno puede ser minimizado con un rendimiento superior de las empresas con el desarrollo de acciones innovadoras y en la atuación proativa para en el ambiente internacional.

Cambios significativos en el escenario mundial demuestran que las empresas tienen que plantearse sus modelos mentales tradicionales de gestión y reemplazar modelos antiguos que pueden causar la pérdida de oportunidades o la imposibilidad de actuar en otros entornos empresariales más competitivos y de difícil inserción. (WIND; CROOK, 2004)

Por lo tanto, este artículo se estructura de la siguiente manera: primero se analiza la teoría de la gestión del conocimiento para reflexionar sobre el papel del entorno para una óptima absorción del conocimiento por las empresas de los resultados y de las medidas adoptadas para mejorar el rendimiento. Seguidamente se presenta la teoría de la innovación como un componente clave para el éxito de las empresas y el comienzo de su internacionalización. Despues se analizan modelos de internacionalización y la forma en que deben llevarse a cabo en entornos complejos e innovadores. Finalmente presentamos las consideraciones finales de la relación de las tres teorías trabajadas en el contexto empresarial.

\section{Empresas que Comparten Conocimiento en Entornos de INNOVACIÓN}

El conocimiento es un recurso estratégico que puedeen nuevas ideias y operaciones, sea con un conocimiento prático o teórico. Estas dos dimensiones, tácita y explícita del conocimiento se puede verificar que están relacionadas y promueven la creación de nuevos ideias a través de la relación de las empresas con su entorno interno y externo, y también el desarrollo de un proceso llamado espiral del conocimiento (Figura1). (NONAKA; KROGH; VOEPEL, 2006; NONAKA, I.; VON KROGH, 2009)

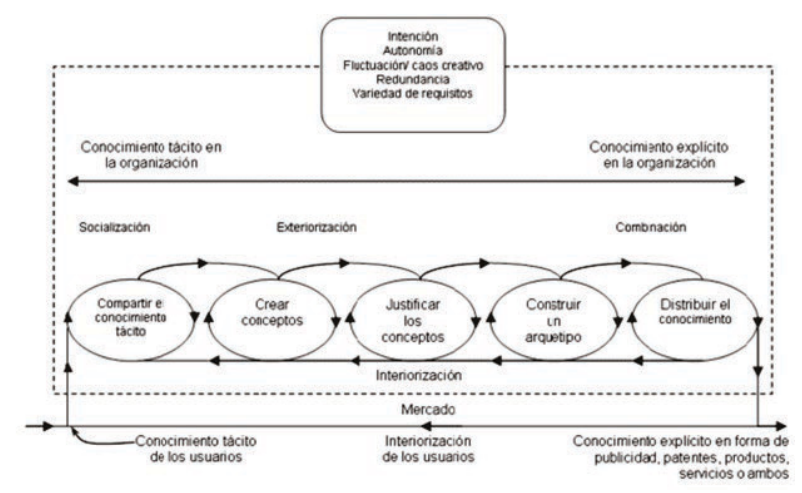

Figura 1: Espiral del conocimiento Fuente: Nonaka y Von Krogh (2009) 
La gestión del conocimiento ha existido siempre en las empresas en sus procesos de codificación, almacenamiento y difusión (ALLEE, 2000; ALAVI; LEIDNER, 2001). La principal diferencia hoy es en la clasificación del conocimiento como un recurso crítico, es decir, un recurso renovable y con valor, $y$ que se fortalece de acuerdo con la comprensión de la necesidad de desarrollar nuevas acciones estratégicas para la innovación empresarial. (HALAWI; ARONSON; MACCARTHY, 2005; MOUSTAGHFIR, 2008; NONAKA; VON KROGH, 2009)

No existe un consenso sobre la gestión de los recursos de conocimiento y su aplicación. Sin embargo, evidencias empíricas del desempeño organizacional demuestran que esa relación existe y que la gestión a través de los recursos críticos pueden mejorar el desempeño de las empresas (GOLD, 2001; ADAMS; LAMONT, 2003, KALLING 2003; DARROCH, 2005; ZACK; MCKEEN; SINGH, 2009). Gold (2001), por ejemplo, señala que en la nueva economía la capacidad de las organizaciones está en el valor económico de sus recursos del conocimiento, tales como los canales de distribución y de información y en las asociaciones empresariales. Esa perspectiva establece que la dificultad de los administradores es mejorar el rendimiento de sus empresas no sólo en utilizar los programas de gestión del conocimiento.

Además, Darroch (2005) investigó la gestión del conocimiento como un mecanismo de coordinación, y a partir de un estudio empírico demuestra que existe mayor rendimiento en las empresas que utilizan gestión del conocimiento para la gestión eficiente de sus recursos y mejor desarrollo de la innovación.

Zack, McKeen y Singh (2009) analizaron a 189 directivos de empresas de servicios y fabricación e identificaron los recursos de conocimiento relacionados con el desempeño organizacional, y seus resultados concluyen que la mayoría de las empresas pueden aumentar el éxito de sus iniciativas con la gestión del conocimiento a través de prácticas de gestión específicas y directas durante su implementación. Por lo tanto, los estudios presentados concluyen que hay una relación directa entre la gestión del conocimiento y el mayor rendimiento de la innovación de las empresas.

También se considera que la aplicación de buenas prácticas en las empresas puede ayudar a los gestores a promover una mejor gestión del conocimiento de sus negocios globales debido al uso de procesos desarrollados y optimizados en otros entornos, y por lo tanto, los procesos que estuvieron en situaciones pasadas pueden ayudar a mejorar el desempeño de las empresas. (LEE; CHOI, 2003; NONAKA; KROGH; VOELPEL, 2006; MARQUÉS; SIMON, 2006; GONÇALO; BORGES, 2010)

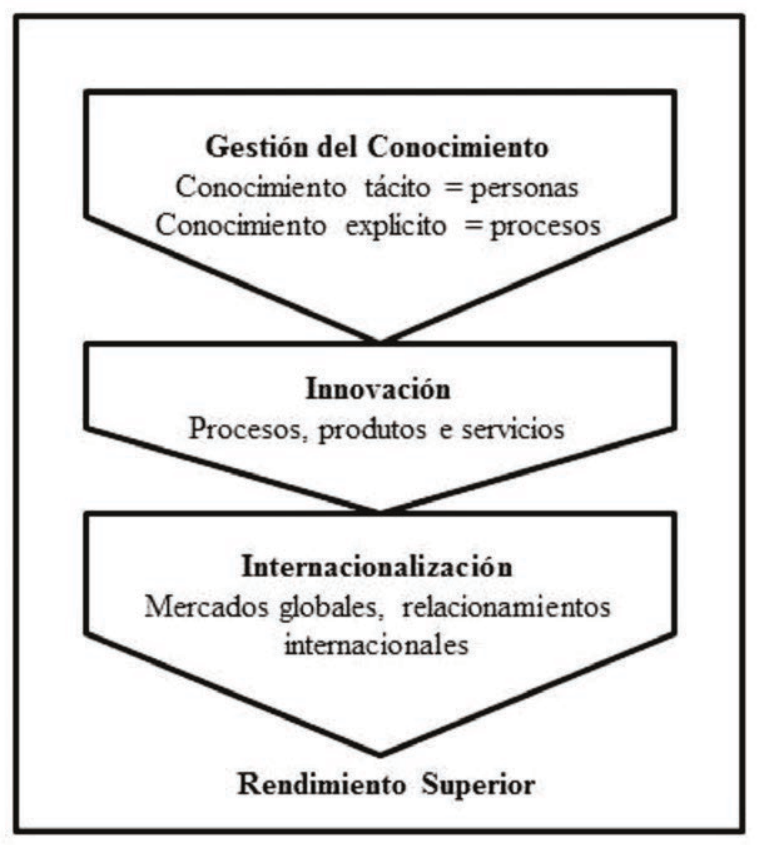

Figura 2: Factores esenciales para el rendimiento superior Fuente: Elaborada por los autores de este artículo

La Figura 2 señala que es importante que las empresas tengan estrategias definidas y están listas para las operaciones internacionales en un entorno dinámico y volátil. La empresa aún tendrá que desarrollar un gran conocimiento de los mercados mundiales, conocer las nuevas tecnologias, culturas y superar las dificultades, tanto en el extranjero como en su país de origen, haciendo adecuación competitiva y de sostenibilidad para la actuación en el mercado internacional.

La gestión del conocimiento es un conjunto de estrategias utilizadas para crear, adquirir, compartir y aplicar los activos del conocimiento que tiene como objetivo establecer rutinas que pueden ayudar a todos los gestores a tener las informaciones necesarias para la generación de ideas, para la solución de problemas y para la toma de decisiones. También es una acción que puede agregar valor para ayudar los gestores a crear nuevas práticas, propagarlo por toda la organización e incorporar el nuevo conocimiento en la innovación 
de productos, servicios y processos. (NONAKA; VON KROGH, 2009; VASUDEVA; SPENCER; TEEGEN, 2013; MORÉ et al., 2014)

Además, la aplicación de la gestión del conocimiento provoca un cambio de cultura en la organización y esto es muy difícil de aceptar tanto por los individuos como por las organizaciones. Generalmente, hay una preocupación para que las funciones de producción y de gestión estén integradas y para que la organización se convierta en un conjunto de grupos interdependientes $e$ interrelacionados que buscan objetivos comunes. (WISCHNEVSKY; DAMANPOUR; MENDEZ, 2010; PADILLA-PÉREZ; GAUDIN, 2014)

Por lo tanto, el conocimiento es un conjunto de experiencias, valores e información que ayuda a la empresa y a los individuos a evaluar sus acciones y tener nuevas experiencias. Las empresas pueden adquirir un potencial infinito de creación de conocimiento una vez que sus miembros tienen la oportunidad de pensar, aprender y hablar unos con los otros continuamente para compartir sus conocimientos. (ESTRIN; BAGHDASARYAN; MEYER, 2009; FILIOU; GOLESORKHI, 2014)

Sabemos que el conocimiento solamente puede ser desarrollado por los individuos de la organización. Por esto, tenemos que fomentar y estimular a las empresas y las personas a ser creativas y tener las condiciones necesarias para el acceso a la información en la creación constante de nuevos conocimientos. Una manera excelente de estimular la gestión del conocimiento es a través de la capacitación y la formación continua de las personas, donde la universidad tiene un papel muy importante en este proceso porque ella puede dar formación sobre grupos de trabajo en las empresas y ayudar en el desarrollo organizacional. (ANDREU-ANDRÉS; GARCÍA-CASAS, 2014)

Hay también em los estudios de la gestión del conocimentos los conceptos que capturam el proceso de Capacidad Absorptiva, en que tenemos, por ejemplo, las dimensiones de reconocimiento, asimilación y explotación; cuatro dimensiones que compreende la capacidad absorptiva en potencial y de realización; tres dimensiones en el proceso de exploración, de transformación y de explotación; y las dimensiones de reconocimiento, aquisición, asimilación o transformación y explotación. (COHEN; LEVINTHAL, 1990;
ZAHRA; GEORGE, 2002; LANE; KOKA; PATHAK, 2006; TODOROVA; DURISIN, 2007)

Por otra parte, las teorías institucionales señalam cómo el ambiente externo de una empresa interactua con su capacidad absorptiva afectando su rendimiento de la innovación (LICHTENTHALER; LICHTENTHALER 2009). La sostenibilidad y desarrollo de las empresas a nivel nacional tiena diferencias de su competitividad en comparación con la atuación internacional, una vez que las empresas se esfuerzan aún más por aprovechar los centros internacionales de excelencia, compensando las deficiencias de los recursos del país de origen o por la poca capacidad de desarrollar nuevos productos.

Como las diferencias de instituciones informales aumentan en alianzas internacionales, las empresas deben estar cada vez más atentas, conscientes y perceptivas del cómo estas diferencias pueden crear obstáculos en el intercambio de conocimientos en estos casos. Sin embargo, aumentar la consciência que hay en las diferencias podría facilitar la agilidad e interés de invertir tiempo para la realización de alianzas específicas necesarias para gestionar de forma explícita y directa las diferencias. (GONZALES; MARTINS; TOLEDO, 2009)

Sin embargo, es importante también pensar en las acciones estratégicas que pueden ser implementadas para optimizar la gestión del conocimiento, la innovación y la consecuente internacionalización de las empresas que están en un entorno de vocación innovadora (Figura 2). El conocimiento tácito en la rutina de los empleados tiene que ser mejor desarrollado para convertirse en conocimiento explícito que sea fácil de comprender y puede convertirse en mejores prácticas de gestión para que otras personas y empresas quieran seguir, generando flujo permanente de innovación de procesos, servicios y productos y mejora de el desempeño organizacional.

\section{Innovación como Proceso de Cambio y Mejora Contínua}

Las innovaciones ofrecen oportunidades para que las empresas actúen con mayor precisión en espacios de mayor necesidad en el mercado y ofrezcan productos y servicios que hasta ahora no existían y no 
podían ser aceptados por los clientes (PAVITT, 2004). Una gran velocidad del proceso de innovación también es necesaria para reducir el ciclo de vida de los productos o servicios y el tiempo de desarrollo es cada vez menor debido a la mayor demanda de los clientes por servicios instantáneos. (OCDE, 2005)

Otros puntos destacados del proceso de innovación son la intensidad de la competencia, la rápida globalización y los cambios en el área de tecnología de la información que hacen inevitable la innovación empresarial como una manera de capturar nuevas oportunidades a través del desarrollo de productos y servicios innovadores para mercados que están en crecimiento. (LOBIANCO; RAMOS, 2004; KUBOTA, 2009)

La innovación se relaciona con la investigación, las oportunidades o la tecnología de desarrollo. También las capacidades de aprendizaje para implementar nuevas formas de gestión de las organizaciones y la aparición de nuevos aspectos sociales y económicos están presentes en el proceso de innovación; por ejemplo, la creación de un entorno para un mejor funcionamiento de las redes organizacionales. (LOBIANCO; RAMOS, 2004; ALVARENGA NETO, 2004; BJÖRKLUND; KALLING; ÅKERLINDH, 2008; KUBOTA, 2009)

En esta perspectiva se comprende la importancia de la innovación en contextos donde existe la participación integrada de las empresas, aunque se necesitaría mejorar el proceso de creación y desarrollo de nuevos productos. Gomes, Machado y Giotto (2009) presentan un trabajo sobre las características de los estudios de innovación presentados en congresos científicos en Brasil con artículos publicados desde 1997 hasta 2009 y que fueron aplicados en diferentes entornos de investigación. En muchos de los estudios presentados es posible verificar que la innovación se relaciona con el conocimiento porque puede crear nuevas oportunidades que combinan diferentes informaciones.

Por lo tanto, el resultado de este proceso es la búsqueda de nuevas tecnologías, nuevos mercados o la supervisión de la competencia, lo que se transforma progresivamente en una estrategia de gestión de gran potencial. Dicha estrategia se desarrolla en situaciones específicas identificadas en un lugar concreto y se comprueba que el mejor desempeño está garantizado por la capacidad de utilizar bien el conocimiento.

El desarrollo de nuevos productos o servicios es una capacidad muy importante de las empresas por el entorno empresarial de los negocios, que cambia constantemente. Las empresas deben estar preparadas para reaccionar ante la creación de nuevos productos y servicios antes que los competidores. (TIDD; BESSANT; PAVITT, 2008; MORÉ, et al., 2014)

En un estudio realizado en 258 empresas españolas, González y Nieto (2007) fueron capaces de identificar los procesos y los recursos que las empresas se apropian para buscar resultados innovadores, como las patentes, los secretos comerciales o las actividades de mejora continúa. Los resultados presentados confirman que las empresas que utilizan el conocimiento explícito, por ejemplo, el que está en los libros o manuales de enseñanza, tienen mecanismos de defensa como las patentes. Sin embargo, las empresas que optan por el conocimiento tácito, que está en la mente de las personas, tienden a depender principalmente del secreto comercial.

Ante esto, los procesos de innovación deben implicar la explotación de oportunidades de nuevos productos, procesos o servicios basados en el avance de la técnica, la búsqueda de nuevos mercados o una combinación de ambos. La innovación es esencialmente un proceso de adaptación a estos mercados y se puede reflejar en iniciativas de internacionalización o en la búsqueda de nuevos nichos de especialización. (CAÑAVATE, 2009; GUAN; CHEN, 2010)

Guan y Chen (2010) presentan los tipos de activos de una empresa (Figura 3) donde los activos y los factores se subdividen en tangibles e intangibles.

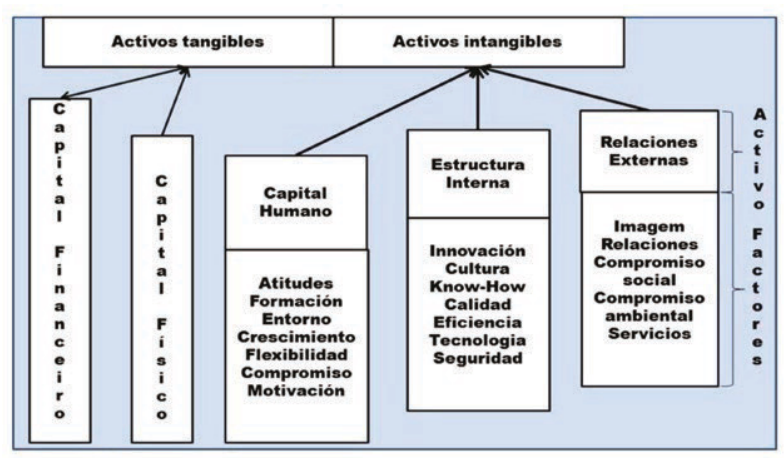

Figura 3: Tipos de Activos

Fuente: Guan y Chen (2010)

Destacamos en la Figura 3 que los activos pueden ser factores tangibles e intangibles, igual que los productos o servicios y procesos, y que el sistema de creación e innovación puede trabajar con ambos tipos 
de recursos para obtener los mejores resultados posibles ante la innovación. Sin embargo, esta secuencia no es fácil. Por ejemplo, aunque el proceso de innovación esté en marcha, a menudo las personas podrían no saber exactamente qué está pasando porque se refleja en factores intangibles, y los gerentes o empleados podrían desanimarse y no dar continuidad al proceso de innovación organizacional.

Otros estudios recientes de la innovación se refieren a dos tipos básicos de la innovación: innovación cerrada e innovación abierta. La mayoría de los avances tecnológicos que se presentan entre los siglos XIX y XX se produjeron en la forma conocida como innovación cerrada, modelo en que las grandes empresas desarrollaron sus ideas a partir de sus actividades de I+D. (CHESBROUGH, 2006; CHESBROUGH; APPLEYARD, 2007)

La gestión de la innovación cerrada fue habitual hasta mediados del siglo XX, principalmente por la falta de recursos en las universidades y del Estado a la hora de apoyar la investigación. Entonces, los proyectos empresariales consistían en iniciativas en las que las empresas estuvieron invirtiendo internamente en el desarrollo de nuevos productos, servicios o procesos, y para ser competitivas en el mercado buscaban alternativas para mantener su investigación en el tiempo y que la propiedad intelectual estuviera bien protegida para evitar el espionaje industrial. (CHESBROUGH, 2006; CHESBROUGH; APPLEYARD, 2007; CHESBROUGH; SCHWARTZ, 2007; CHIARONI; CHIESA; FRATTINI, 2010)

A través de los años, y con la innovación tecnológica, surge el concepto de innovación abierta. Su aparición es un nuevo paradigma de la gestión de la innovación en el siglo XXI, en un contexto marcado por las fuerzas competitivas del mercado y por los constantes cambios en las organizaciones, aunque el conocimiento se convierte en el principal activo estratégico, haciendo que las empresas sean abiertas para que colaboren con otros grupos de interés y exploren nuevos conceptos que influyan en el proceso de innovación continua.

Las empresas globales han de averiguar cuáles son los caminos, capacidades y recursos para el proceso de la innovación más adecuados a su estructura organizacional. Sin embargo, un concepto general es conocido y aceptado por todos: es preciso innovar.
Pensar en un modelo de innovación abierta, que sucede en los parques científicos o distritos de innovación, parece ser lo más adecuado, sobre todo cuando asociamos los procesos de gestión del conocimiento y la internacionalización de las empresas.

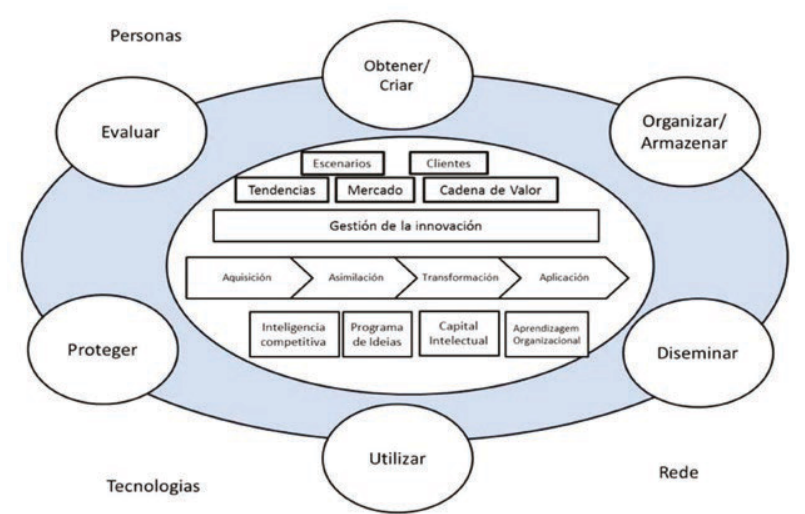

Figura 4: Gestón del conocimento para la inovación abierta Fuente: Elaborada por los autores de este artículo

Para averiguar cuales son los mejores caminos, las empresas también necesitan de competencias esenciales, siendo tres las competencias organizacionales que deben ser desarrolladas por las empresas con objetivo de maximizar el valor de sus tecnologias y hacerlas más competitivas: interacción del sistema; integración del sistema con otros sistemas; y viabilización técnica. (BIANCOLINO; RICCIO; MACCARI, 2011)

La gestión y los procesos de cada empresa deben ser analizados, identificados y desarrollados a partir de los recursos estratégicos que deben ser absorbidos por el mercado (OCDE, 2005; KIRSCHBAUM, 2010). Una vez presentados los fundamentos que representa la innovación, como el estudio de la innovación abierta, es preciso también investigar como la creación de un ambiente organizacional puede ayudar a mejorar el potencial de innovación de las organizaciones, es decir, la creación de un entorno que ayude a aumentar la capacidad de innovación de las organizaciones. Este es el siguiente tema de análisis en este trabajo.

\section{La Capacidade de Innovación en las EMPRESAs como Respuesta a los Cambios del Entorno}

La capacidad de innovación se utiliza por lo general como una forma de medir el grado de novedad de 
alguna innovación de las organizaciones, mejorando la capacidad o la propensión de la empresa para innovar o desarrollar nuevos productos. (CALANTONE; CAVUSGIL; ZHAO, 2002; ANDREASSI; SBRAGIA, 2004; WISCHNEVSKY; DAMANPOUR; MÉNDEZ, 2010)

Aún existen diferencias importantes entre los conceptos de innovación e innovatividad. La innovación es un resultado eventual, un producto o proceso, que se expresa en el contexto intrínseco y extrínseco a la organización en atención a su influencia sobre el conocimiento. La innovatividad es una red de capacidades, actitudes y recursos materiales y tecnológicos, en sintonía con redes de personas e instituciones organizadas con una visión de crecimiento y desarrollo de sí mismas, con actitudes orientadas por la apertura de posibilidades, la cooperación, y la buena calidad de procesos y productos. (BUSTAMANTE; MALDONADO, 2005)

Gómez (2006) aborda la importancia de que a cada día se deben atender las demandas de una organización, hacer que ésta dirija sus esfuerzos y recursos a la búsqueda de nuevos métodos, enfoques y modelos para satisfacer esas demandas. Esto conduce a que ya no sólo sea importante la innovación, sino que debe extenderse a la innovatividad, es decir, a la conformación de redes de innovación productivas.

La innovatividad es considerada una de las principales formas de lograr una ventaja competitiva en las empresas (HULT; KETCHEN, 2001; HULT; HURLEY; KNIGHT, 2004; TAJEDDINI; TRUEMAN; LARSEN, 2006). Es un medio para diferenciar una empresa de la otra, lo que puede hacer que el cliente decida sobre la compra de un producto o no, y también determinar el éxito de un mejor desempeño de la organización.

La innovatividad refleja la tendencia de las organizaciones de buscar nuevas ideas y desarrollar nuevos productos y servicios (LUMPKIN; DESS, 1996). La capacidad de innovación de la organización es una característica que forma parte de la cultura de la empresa y refleja su deseo de lograr nuevas oportunidades y generar una mayor capacidad de innovación. (SUBRAMANIAN, 1996; HURLEY; HULT, 1998).

En la literatura son pocos los estudios que se han elaborado sobre las formas de medirla y también son pocos los estudios que se refieren a la capacidad de innovar de las empresas. (CALANTONE; CAVUSGIL; ZHAO, 2002)
En este punto, es importante mejorar la comprensión de estos dos conceptos. La innovación puede ser entendida como el resultado final, es decir, un nuevo producto o servicio que se ha desarrollado. Sin embargo, la innovatividad es la variable que representa la orientación, la proactividad de la empresa o la inclinación a la innovación. Una definición objetiva sería la disposición cultural de la empresa, inclinación o propensión para probar nuevas ideas y dejar atrás viejos hábitos. (HURLEY; HULT, 1998; HULT, 2004; MENGUC; AUH, 2010)

En cuanto a la disposición de la empresa en la búsqueda de la innovación, tenemos la idea de crear un entorno que ayude en la creación del conocimiento de las empresas, también llamado de entorno ba. El entorno ba es el context ideal para las relaciones comerciales y la creación del conocimiento de las empresas. Se entiende que por medio del ambiente ba el conocimiento puede nacer de la interacción intra o inter empresas en diferentes ecosistemas de negocios. (NONAKA; VON KROGH, 2009)

En esta línea de razonamiento, el entorno ba se acerca al concepto de innovatividad, que también implica la necesidad de las empresas de ser más proactivas, siguiendo ciertas rutinas y procesos para aprovechar nuevas oportunidades. (MENGUC; AUH, 2010)

Damanpour (1991) y Gopalakrishnan y Damanpour (2000) entienden la innovación como sinónimo de una rutina de innovaciones, de cualquier naturaleza, adoptadas por empresas en un período determinado. Una definición similar es presentada por Hurley y Hult (1998) y Hult, Hurley y Knight (2004) que definen la innovación como un precursor cultural que hace que la empresa tenga una conducta orientada a la creatividad y la capacidad de adaptación. McDonald (2003) define la innovación como la voluntad y la capacidad de la empresa para adoptar nuevas tecnologías, procesos e ideas, ofreciendo productos únicos e inovadores ante sus competidores.

Menguc y Auh (2010) afirman que para que una empresa sea innovadora debe adoptar una nueva mentalidad de compartir y difundir información y conocimiento por toda la organización, activos que son intangibles, complejos y difíciles de medir.

Siendo la innovación intangible y de difícil imitación por los competidores, las empresas pueden tener con esto una ventaja competitiva sostenible. Por ello, 
tienen que reorganizar constantemente sus recursos para desarrollar las fuerzas competitivas que pueden crear barreras para nuevos competidores. (REED; DEFILLIPI, 1990)

En una investigación realizada en Shanghai con empresas que competían entre sí, Menguc y Auh (2010) analizarón que el éxito de las empresas está relacionado con la capacidad de innovación y el alto nivel de orientación al mercado que tiene, así como el clima y la cultura organizacional orientada al mercado externo.

Presentado el marco conceptual de la gestión del conocimiento e innovación, y cómo estas perspectivas teóricas se posicionan en entornos organizacionales integrados, se aborda la teoría de la internacionalización en sus diferentes perspectivas teóricas.

\section{LA INTERNACIONALIZACIÓN DE LAS EMPESAS Y SU RELACIÓN CON LA Gestión del Conocimiento y la InNOVACIÓN}

El uso general y disconexo del concepto de internacionalización es un problema en la literatura. Es común hablar que la internacionalización se caracteriza por el movimiento de las organizaciones fuera de su país de origen o de la ejecución de operaciones internacionales (exportación). (WELCH; LUOSTARINEN, 1993)

El tema de la internacionalización ha sido muy debatido por los académicos. Por esto, encontramos distintos enfoques económicos (BUCKLEY; CASSON, 1976; DUNNING, 1998, 2001); los modelos en forma de U (modelo de Uppsala) (JOHANSON; WIEDERSHEUM-PAUL, 1975; JOHANSON; VAHLNE, 1977; 2003; JOHANSON; VAHLNE, 2013; HOHENTHAL; JOHANSON; JOHANSON, 2014); y los modelos que se refieren a las diferentes etapas de internacionalización (relacionados con la innovación). (BILKEY; TESAR, 1977; CAVUSGIL, 1980)

Se sabe que la internacionalización supone altos costes directos y muchas dificultades para el proceso de producción, como la adecuación de distintos procedimientos y técnicas. A pesar de los riesgos de la internacionalización, puede ser favorable para el desarrollo de la empresa que busca ventajas competitivas. (GATTAI; MOLTENI, 2007)
El modelo comportamental de la escuela de Uppsala se acerca a los aspectos teóricos de la gestión del conocimiento; esa teoría también es conocida como modelo dinámico de aprendizaje. Uppsala se desarrolló en la década de 1970 por Johanson, que estudió organizaciones suecas: Sandvik, Atlas Copco, Facit y Volvo. Este autor analizó la forma en que estas empresas actuaban en los mercados internacionales, identificando algunas características comunes. Esas características fueron nombradas de distancia psíquica, esto es, que cuanto mayor es el grado de conocimiento de la organización sobre el mercado más tiende a invertir recursos en el mismo. De acuerdo con este modelo, el proceso de internacionalización se desarrolla en cuatro etapas principales:

a) Actividades de exportación irregulares;

b) Actividades de exportación por medio de representantes;

c) Actividades de exportación a través de una subsidiaria de ventas establecida en los mercados extranjeros;

d) Establecimiento de una estructura de producción o fabricación en los mercados extranjeros.

Johason y Vahlne (1977, 1990, 1992, 2003) han mejorado el concepto de la escuela de Uppsala de forma que las empresas pudieran definir mejor su proceso de internacionalización con variables del conocimiento y mayor compromiso con el mercado. En este caso el conocimiento se refiere a las informaciones que se tiene del mercado que está buscando. Además, el compromiso se relaciona con los fondos invertidos en ese mercado. Para Andersen (1993) la escuela de Uppsala es considerada una importante corriente literaria en los estudios de los negocios internacionales.

Diferente de la escuela de Uppsala, que establece el proceso de internacionalización con las presiones sufridas por las empresas en la búsqueda de nuevos mercados, el paradigma ecléctico estudia factores generales que hacen a una empresa buscar un mercado internacional. Dunning (1998) es el principal autor de esta corriente, y analizo qué hace a una organización lograr buenos resultados en su proceso de globalización, aunque para esto es necesario estudiar factores importantes de ese nuevo mercado, por ejemplo, problemas con la propiedad intelectual o de ubicación. 
Dunning (2001) reconoce que el paradigma ecléctico no es el único modelo para analizar la dinámica de la producción internacional. No obstante, puede ayudar a explicar por qué una inversion realizada por una empresa en un país extranjero puede ser diferente considerando momentos o situaciones distintas.

Molero y García (2008), por su parte, analizaron los reflejos de estrategias innovadoras de empresas multinacionales de la industria manufacturera española en los países que operan, llamados países intermedios. La capacidad de integrar las fuentes internas y externas de conocimiento, recursos humanos cualificados y disponibilidad de fondos fueron factores utilizados para evaluar el desempeño de las empresas.

El desarrollo de la competencia personal también es factor clave para el éxito organizacional, y en una investigación aplicada es posible identificar procesos, recursos y situaciones que ayudarían a evaluar la capacidad de innovación de una persona en el ámbito académico. (WATTS et al., 2012)

El modelo de la escuela de Uppsala muestra varias etapas para el proceso de internacionalización de las organizaciones. Las empresas tienen un proceso de internacionalización que a menudo se adelanta a los mercados internacionales, aunque también pueden desarrollar su relación con esos mercados, teniendo un aumento de la complejidad de su estructura organizacional (JOHANSSON y VAHLNE, 1977; RAMAMMURTI; SINGH, 2010). Estas etapas pueden verse en la Tabla 1.

Tabla 1: Etapas de la internacionalización

\begin{tabular}{|c|c|c|}
\hline EtAPA & ReLACIÓN & OBSERVACIÓN \\
\hline 1 & $\begin{array}{l}\text { No hay } \\
\text { exportaciones } \\
\text { regulares }\end{array}$ & $\begin{array}{l}\text { No hay ningún compromiso entre } \\
\text { la empresa y el mercado y, por } \\
\text { lo tanto, no hay necesidad de un } \\
\text { canal regular de comunicación. }\end{array}$ \\
\hline 2 & $\begin{array}{l}\text { Exportar a través } \\
\text { de agentes }\end{array}$ & $\begin{array}{l}\text { La empresa cuenta con un } \\
\text { canal regular de información del } \\
\text { mercado que obtiene información } \\
\text { relevante acerca de los factores } \\
\text { que influyen en sus ventas. }\end{array}$ \\
\hline 3 & Filial de ventas & $\begin{array}{l}\text { Hay un canal de información entre } \\
\text { la empresa y el mercado dando a } \\
\text { la compañía la capacidad de dirigir } \\
\text { el tipo y la cantidad de información } \\
\text { que necesita en el mercado. }\end{array}$ \\
\hline 4 & $\begin{array}{l}\text { Unidades } \\
\text { productivas o } \\
\text { de fabricación }\end{array}$ & Mayor compromiso de los recursos. \\
\hline
\end{tabular}

Fuente: Johanson e Wiedershem (1975)
Por otra parte, el modelo I-model el proceso de internacionalización se entiende como una secuencia de fases aplicada a los mercados cuya distancia psíquica sea mayor, y cada etapa genera una nueva comprensión para la empresa. (ANDERSEN, 1993)

La globalización económica y el aumento del flujo de bienes, servicios o capitales implica no sólo nuevas oportunidades sino también nuevos retos para las empresas. La relación de las empresas debería ser mayor en el mercado internacional para tener más oportunidades. Muchas empresas, especialmente PYME, no aprovechan al máximo el potencial del mercado para la exportación debido a la falta de motivación, de capacidades o de recursos financieros o humanos, y por lo tanto el trabajo de las empresas en parques científicos puede mejorar su rendimiento y ser una buena alternativa para la sostenibilidad de la organización, aunque los conjuntos de servicios pueden ser creados y ofrecidos a través de iniciativas públicas o privadas destinadas a ayudar a las empresas a superar los obstáculos. (RAMAMMURTI; SINGH, 2010; FREIXANET, 2012)

El Modelo de Uppsala dice que el aprendizaje se adquiere a través de la experiencia de los gestores (Johanson; WIEDERSHEM, 1974), y por lo tanto, la comprensión de los factores que promueven la internacionalización desde la perspectiva de la gestión del conocimiento y de la innovación es un elemento innovador para los estudios organizacionales.

\section{Consideraciones Finales}

Este artículo se propone relacionar la gestión del conocimientos y la innovación como factores estratégicos para la internacionalización de las empresas, tenendo uma gran contribuición teórica en la identificación de los elementos-claves para la participación activa de las empresas con mercados globales y competitivos.

La gestión del conocimiento y la innovación en entornos complejos como solución para los procesos de internacionalización son factores decisivos para el desarrollo de nuevos productos y servicios y la búsqueda de nuevas oportunidades, aunque la innovación a menudo debe basarse también en el conocimentos de las mejores prácticas y generar respuestas rápidas a los cambios em la búsqueda de nuevos mercados. 
(ZACK; MCKEEN; SINGH, 2009; WISCHNEVSKY; DAMANPOUR; MENDEZ, 2010; VASUDEVA; SPENCER; TEEGEN, 2013; MORÉ et al., 2014).

El ejemplo de los parques científicos de innovación o ambientes similares puede ayudar a entender el nexo que existe entre las teorías presentadas en este artículo. La Asociación Internacional de Parques Científicos y Áres de Innovación (IASP) define parque científico como una organización gestionada por profesionales especializados cuyo reto es mejorar cada día la comunidad y promover una cultura de innovación y de competitividad para las empresas y las instituciones basadas en conocimiento. El parque debe estimular y gestionar el intercambio de conocimientos y tecnologías entre universidades, instituciones de $\mathrm{I}+\mathrm{D}$, empresas y mercado y facilitar la creación y el crecimiento de empresas a través de la incubación o procesos de spin-offs, lo que genera un valor adicional a los servicios a través de instalaciones que ayudan en el desarrollo organizacional. (CCIP, 2008)

Hay diferencias en la sostenibilidad y la persistencia de las empresas a nivel nacional de su competitividad y en el camino para la colaboración internacional, una vez que las empresas se esfuerzan por aprovechar los centros internacionales de excelencia, o también para compensar las deficiencias de los recursos del país de origen o la poca capacidad de desarrollar nuevos productos. De hecho, los impulsores de la I+D para la internacionalización son similares. (ANDERSEN, 1993; CCIP , 2008; CAÑAVATE, 2009; AUTIO, 2014)

El aumento de la internacionalización en alianzas amplía la variedad de conocimientos accesible para las empresas. Además, el aumento de la riqueza de oportunidades para la innovación hace que los socios aprovechen los conocimientos con mayor diversidad, criando nuevas tecnologías y tendencias para la demanda. (AUTIO, 2014)

Del mismo modo, cada vez más sistemas informales de gestión del conocimiento proporcionan una mayor comprensión para la definición del problema y su solución, como también un conjunto más rico con diversidad de nuevos sistemas de gestión e integración del conocimiento que podrían beneficiar las empresas con nuevas ideas.

Con respecto a las etapas de la empresa en su proceso de internacionalización (JOHANSON; WIEDERSHEIM $\square$ PAUL, 1975), la Figura 5 demuestra que la empresa debe evaluar la relación de la gestión de sus conocimientos con los recursos de innovación necesarios para buscar una etapa superior y que le permitirá apuntar mejores resultados. Los procesos de aquisición, asimilación, transformación y aplicación de conocimientos (TODOROVA; DURISIN, 2007) requerirán mayores esfuerzos y recursos en etapas que la empresa está trabajando o buscando una relación internacional mayor.

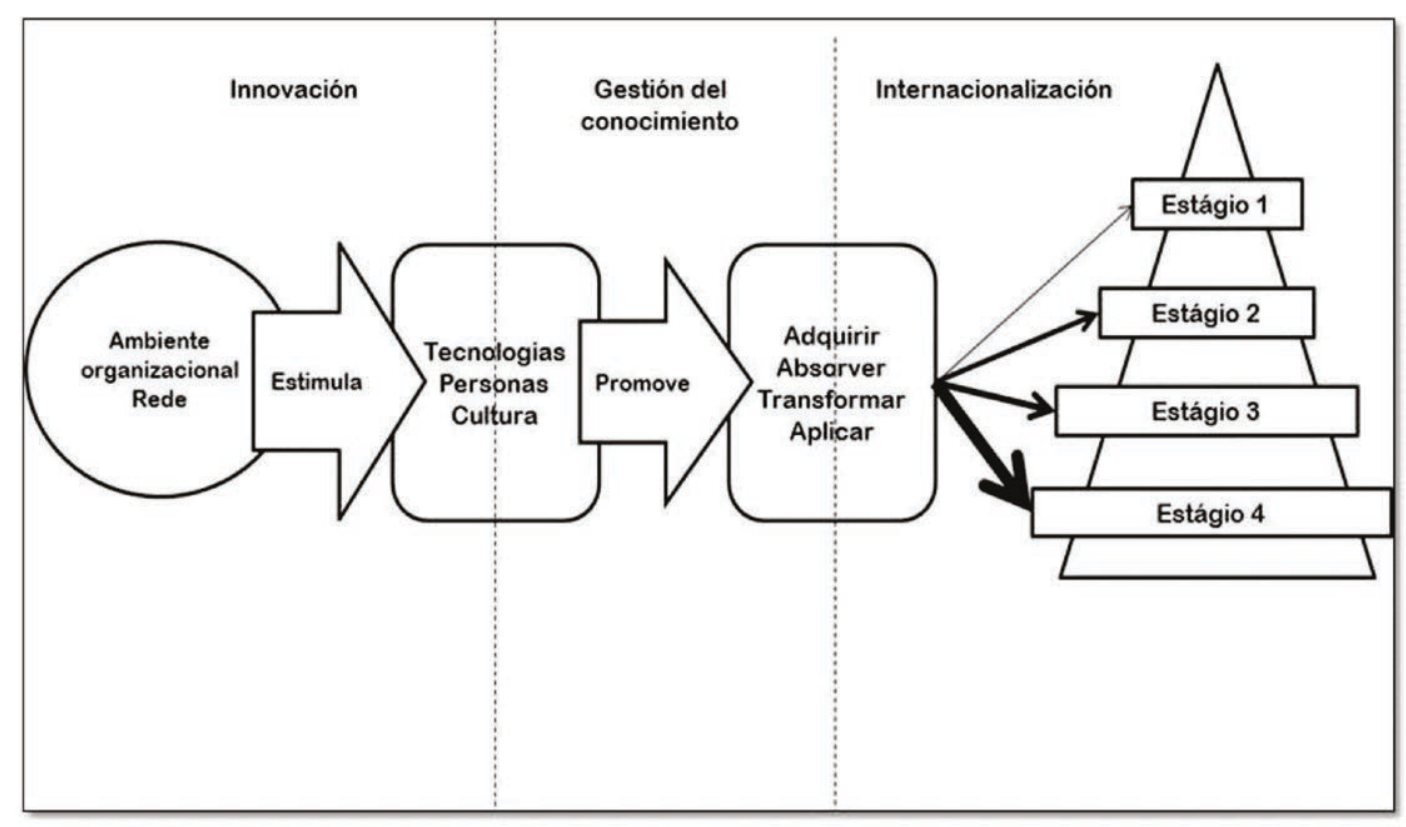

Figura 5: La búsqueda de la internacionalización

Fuente: Elaborada por los autores de este artículo 
En el caso analizado de la Escuela de Uppsala, que trabaja con cuatro etapas para la acción internacional: no realización de actividades regulares de exportación; exportar con intermediarios; establecimiento de una filial en el extranjero; y de producción en el extranjero, con instalaciones de fabricación, la necesidad de un mayor conocimiento de los mercados internacionales, en el caso de la etapa 4, hay prioridad para nuevas informaciones sobre el mercado, personal con formación cualificada y la búsqueda de nuevas relaciones comerciales que permitan las empresas obtener mayor retorno financiero en el exterior, y para ellas, su localización en el entorno nacional podría facilitar la obtención de recursos y agilizar los procesos burocráticos, pudiendo obtener beneficios por estar asociada a una red de parques, por ejemplo.

En la etapa primaria de la internacionalización, las empresas tienden a guiarse por otras empresas, copiar sus caminar, estar orientado en el camino o el copiado de la lógica de otra persona. Sin embargo, la normalización a escala global puede generar burocratización y racionalización (DIMAGGIO; POWELL, 2005), pudiendo ser minimizado a través de alianzas con otras empresas para resolver problemas burocráticos, una vez que un entorno compartido pueda ayudar las empresas en su capacidad absorptiva y búsqueda de innovación.

El caminho percorrido por las empreas en su processo de internacionalización demanda en cada etapa de atuación estrategias y conociminetos específicos, que pueden sofrir modificaciones en cada fase nueva. Esa adecuación es fundamental en la capacidad absorptiva de la empresa para el desarrollo de nuevos conocimientos necesários para permanecer o llegar a diferentes mercados globales.

Por lo tanto, la gestión del conocimiento a través del tácito y explícito presente en los procesos de gestión ayuda a optimizar las actividades de negocio y llevar a cabo las mejores prácticas en empresas cuyo objetivo es la gestión eficiente de la innovación de productos, servicios y procesos de internacionalización, de manera que se puede acceder a los mercados globales con mayor precisión y se generan nuevas relaciones a través de alianzas estratégicas o asociaciones internacionales.

Es importante destacar que estudios señalan que hay una fuerte relación entre las teorías de internacionalización, gestión del conocimiento e innovación en entornos de red (ZAHRA; GEORGE, 2002; ZACK; MCKEEN; SINGH, 2009; YANGA; MOTOHASHIB; CHENC, 2009; VAHLNE; JOHANSON, 2013; PADILLA-PÉREZ; GAUDIN; 2014), y los directivos de las empresas con potencial innovador pueden hacer uso de esta relación cuyo reto es evaluar mejor su organización y proponer soluciones innovadoras que pueden ayudarlos a superar los cambios del mercado.

Teniendo en cuenta la configuración de los parques tecnológicos o científicos esta conexión es más clara y directa debido a la necesidad de los gestores en compartir los conocimientos que hay en sus empresas, innovando y buscando nuevos canales de venta a nivel internacional.

\section{Referencias}

ADAMS, G. L.; LAMONT, B. T. Knowledge management systems and developing sustainable competitive advantage. Journal of Knowledge Management, [S.1.], v. 7, n. 2, p. 142-154, 2003.

ALAVI, M.; LEIDNER, D.E.R. Knowledge management and knowledge management systems: Conceptual foundations and research issues. MIS quarterly, [S.1.], p. 107-136, 2001.

ALLEE, $V$. Reconfiguring the value network. Journal of Business strategy, [S.1.], v. 21, n. 4, p. 36-39, 2000.

ALVARENGA NETO, R. C. D. A construção do conceito de gestão do conhecimento: práticas organizacionais, garantias literárias e o fenômeno social. Reuna, Belo Horizonte, v. 9, n. 2, p. 57-74, 2004.

ALVAREZ, I.; MOLERO, J. Technology and the generation of international knowledge spillovers: An application to Spanish manufacturing firms. Research Policy, [S.1.] v. 34, p. 1.440-1452, 2005.

\section{AMATO, J. N. Redes de cooperação produtiva e}

clusters regionais: oportunidades para as pequenas e médias empresas. São Paulo: Atlas, 2000.

ANDERSEN, O. On the internationalization process of firms: a critical analysis. Journal of international business studies, [S.1.], p. 209-231, 1993. 
ANDREASSI, T.; SBRAGIA, R. Relações entre indicadores de PyD e de resultado empresarial. Revista de

Administração, São Paulo, v. 37, n.1, p. 72-84, 2002.

ANDREU-ANDRÉS, M. A.; GARCÍA-CASAS, M. La evaluación de la participación en equipos de trabajo universitarios (Assessment of participation in higher education team working activities). Working Papers on Operations Management, [S.1.], v. 15, n. 1, p. 1-14, 2014.

AUTIO, E. Entrepreneurial innovation: The importance of context. Research Policy, [S.1.], v. 4, p. 31.097-31.108, 2014.

BERRY, H.; GUILLEN, M. F; ZHOU, N. An institutional approach to cross-national distance. Journal of International Business Studies, [S.1.], v. 41, p. 1.460-1.480, 2010.

BIANCOLINO, C. A.; RICCIO, E. L.; MACCARI, E. A. SOA, ERP II e Competências Organizacionais: traçosde inovação na moderna gestão de TI. Revista de Ciências da Administração, Florianópolis, v. 13, n. 30, p. 146177, maio-ago. 2011.

BJÖRKLUND, M.; KALLING, T.; ÅKERLINDH, K. Specificities vs Enthusiasm: Nine European Case Studies of Commercialization of Innovation. Paper presented at the ICICKM Conference, New York, US, October 8-10, 2008.

BILKEY, W. J.; TESAR, G. The export behavior of smaller-sized Wisconsin manufacturing firms. Journal of international business studies, [S.1.], p. 93-98, 1977.

BUCKLEY, P. J.; CASSON, M. The future of the multinational enterprise. Londres: Macmillan Books, 1976.

BUSTAMANTE, S.; MALDONADO, I. P. de. Capacidad innovativa y organizaciones generadoras de conocimiento.

Revista Omnia, [S.1.], v. 11, n. 1, p. 1-22, 2005.

CALANTONE, R. J.; CAVUSGIL, S. T.; ZHAO, Y. Learning orientation, $\square \mathrm{rm}$ innovation capability, and $\square \mathrm{rm}$ performance. Industrial Marketing Management, [S.1.], v. 31, p. 515-524, 2002.

CAÑAVATE, A. M. La información para la empresa y el sistema de $\mathrm{i}+\mathrm{d}+\mathrm{i}$. el caso de la región valenciana.

Revista General de Información y Documentación, [S.1.], v. 19, p. 121-144, 2009.
CAVUSGIL, S. T. On the internationalization process of firms. European Research, v. 8, n. 6, p. 273-281, 1980.

CCIP. Committee on Comparative Innovation Policy: Best Practice for the 21st Century. Understanding Research, Science and Technology Parks: Global Best Practice: Report of a Symposium. Copyright (C) National Academy of Sciences. All rights reserved, 2008.

CHESBROUGH, H. W. Open Business Models: How to thrive in the new innovation landscape. Harvard Business School Press, 2006.

CHESBROUGH, H. W.; APPLEYARD, M. M. Open Innovation and Strategy. California Management Review, [S.1.], v. 50, n. 1, p. 57-76, 2007.

CHESBROUGH, H; SCHWARTZ, K. Innovating business models with co-development partnerships. Research Technology Management, [S.1.], v. 50, n. 1, p. 55-59, 2007.

CHEVALLARD, Alessandra; DUCH, Emilià. Theory and development of clusters. Revista Econòmica de Catalunya, Catalunya, n. 64, July, 2014.

CHIARONI, D.; CHIESA, V.; FRATTINI, F. Unraveling the process from Closed to Open Innovation: evidence from mature, asset-intensive industries. RyD Management, [S.1.], v. 40, n. 3, p. 222-245, 2010.

COHEN, W. M.; LEVINTHAL, D. A. Absorptive capacity: a new perspective on learning and innovation.

Administrative Science Quarterly, [S.1.], v. 35, n. 1, p. 128-152, 1990.

COLEMAN, J. S. Foundations of social theory. Cambridge, England: Harvard University Press, 1990.

DAMANPOUR, F. Organizational innovation: A meta-analysis of effects of determinants and moderators. Academy of Management Journal, [S.1.], v. 34, n. 3, p. 555-590, 1991.

DAMANPOUR, F; SCHNEIDER, M. Characteristics of Innovation and Innovation Adoption in Public Organizations: Assessing the Role of Management. Journal of Public Administration Research and Theory, [S.1.], v. 19, p. 495-522, 2009.

DARROCH, J. Knowledge management, innovation and firm performance. Journal of Knowledge Management, [S.1.], v. 9, n. 3, p. 101-115, 2005. 
DAVENPORT, T. H.; PRUSAK, L. Working knowledge: how organizations manage what they know. Boston, EUA: Harvard Business School Press, 1999.

\section{DAVENPORT, T. H.; PRUSAK, L. Conhecimento}

empresarial: como as organizações gerenciam seu capital intelectual. Rio de Janeiro: Elsevier Brasil, 2003.

DIMAGGIO, P. J.; POWELL, W. W. A gaiola de ferro revisitada: isoformismo institucional $e$ racionalidade coletiva nos campos organizacionais. Revista de Administração de Empresa, São Paulo, v. 45, n. 2, p.74-89, 2005.

DUNNING, J. H. Explaining international production. London: Unwin Hyman, 1998.

DUNNING, J. H. The eclectic (OLI) paradigm of international production: past, present and future. International journal of the economics of business, [S.1.], v. 8, n. 2, p. 173-190, 2001.

ESTRIN, S.; BAGHDASARYAN, D.; MEYER, K. E. The impact of institutional and human resource distance on international entry strategies. Journal of Management Studies, [S.1.], v. 46, n. 7, p. 1.171-1.196, 2009.

FILIOU, D.; GOLESORKHI, S. Influence of Institutional Differences on Firm Innovation from International Alliances.

Long Range Planning, [S.l.], v. 1, p. 1-16, 2014.

FREIXANET, J. Export promotion programs: Their impact on companies internationalization performance and competitiveness. International Business Review, [S.1.], v. 21, p. 1.065-1.086, 2012.

GATTAI, V.; MOLTENI, C. Dissipation of Knowledge and the Boundaries of the Multinational Enterprise. Review of World Economics, [S.1.], v. 143, n. 1, p. 1-26, 2007.

GOLD, A. H.; MALHOTRA, A.; SEGARS, A. H. Knowledge management: an organizational capabilities perspective. Journal of Management Information Systems, [S.1.], v. 18, n. 1, p. 185-214, 2001.

GOMES, G.; MACHADO, D. D. P.; GIOTTO, O. T. O Que se Produz de Conhecimento Sobre Inovação? Uma Breve Análise das Características dos Artigos de Inovação Publicados nos Anais do EnANPAD (1997-2009). Revista de Ciências da Administração, São Paulo, v. 11, n. 25, p. 209-228, set.-dez. 2009.
GÓMEZ, J. R. Innovación, innovatividad y políticas educativas Laurus. Red de Revistas Científicas de América Latina, el Caribe, España y Portugal, v. 12, n. 22, p. 301-315, 2006.

GONÇALO, C. R.; BORGES, M. L. Gestão do conhecimento. In: BITENCOURT, Cláudia (Org.). Gestão Contemporânea de Pessoas: novas práticas, conceitos tradicionais. 2. ed. Porto Alegre: Bookman, v. 1, p. 57-77, 2010.

GONZALEZ, R. VD.; MARTINS, M. F.; TOLEDO, J. C. de. Gestão do conhecimento em uma estrutura organizacional em rede. Ciência da Informação, São Paulo, v. 38, n. 1, p. 57-73, 2009.

GONZALEZ, N.; NIETO, M. Appropriability of innovation results: an empirical study in Spanish manufacturing firms. Technovation: International Journal of Technological Innovation, Entrepreneurship and Technology Management, [S.1.], v. 27, n. 5, p. 280-295, 2007.

GOPALAKRISHNAN, S.; DAMANPOUR, F. The impact of organizational context on Innovation adoption in Comercial Banks. Transactions on Engineering Management, [S.1.], v. 47. n. 1. p. 14-25, 2000.

GUAN, J.; CHEN, K. Measuring the innovation production process: A cross-region empirical study of China's high-tech innovations. Technovation, [S.1.], v. 30, p. 113, 2010.

GURNEETA, V.; ZAHEER, A. The embeddedness of networks: institutions, structural holes, and innovativeness in the fuel cell industry. Organization Science, [S.I.], v. 24, n. 3, p. 645-663, 2013.

HALAWI, L.; ARONSON, J.; MCCARTHY, R. Resourcebased view of knowledge management for competitive advantage. The Electronic Journal of Knowledge Management, [S.1.], v. 3, n. 2, p. 75-86, 2005.

HOHENTHAL, J.; JOHANSON, J.; JOHANSON, M. Network knowledge and business-relationship value in the foreign market. International Business Review, [S.1.], v. 23, n. 1, p. 4-19, 2014.

HUANG, K. F. Absorptive capacity and autonomous R\&D climate roles in firm innovation. Journal of Business Research, [S.1.], v. 68, p. 87-94, 2015. 
HULT, G. T. M.; HURLEY, R. F; KNIGHT, G. A. K. Innovativeness: Its antecedents and impact on business performance. Industrial Marketing Management, [S.1.], v. 33, n. 5, p. 429-438, 2004.

HULT, G. T. M.; KETCHEN, D. J. Does Market Orientation Matter? A Test of the Relationship between Positional Advantage and Performance," Strategic Management Journal, [S.1.], v. 22, n. 9, p. 899-906, 2001.

HURLEY, R. F.; HULT, G. T. M. Innovation, market orientation, and organizational learning: An integration and empirical examination. Journal of Marketing, [S.l.], v. 62, p. 42-54, 1998.

JOHANSON, J.; WIEDERSHEIM-PAUL, F. The internationalization of the firmfour swedish cases 1 .

Journal of management studies, [S.1.], v. 12, n. 3, p. 305-323, 1975.

JOHANSON, J.; VAHLNE, J. E. The internationalization process of the firm - a model of knowledge development and increasing foreing market commitments. Journal of international business studies, [S.1.], p. 23-32, 1977.

JOHANSON, J.; VAHLNE, J. Business Relationship Learning and Commitment in the Internationalization Process. Journal of International Entrepreneurship, [S.l.], v. 1, p. 83-101, 2003.

KALLING, T. Organisation-internal Transfer of Knowledge and the Role of Motivation. A Qualitative Study.

Knowledge and Process Management, [S.1.], v. 10, n. 2, p. 115-126, 2003.

KIRSCHBAUM, R. Open innovation in practice.

Research-Technology Management, [S.1.], v. 48, n. 4, p. 24-28, 2010.

KOGUT, B.; SINGH, H. The effects of national culture on the choice of entry mode. Journal of International Business Studies, [S.1.], v. 19, n. 3, p. 411-432, 1988.

KUBOTA, L. C. As KIBS e a inovação tecnológica das firmas de serviços. Economia e Sociedade, Campinas, v. 18, n. 2, p. 349-369, 2009.

LANE, P. J.; KOKA, B. R.; PATHAK, S. The reification of absorptive capacity: a critical review and rejuvenation of the construct. Academy of Management Review, [S.1.], v. 31, n. 4, p. 833-863, 2006.
LEE, H.; CHOI, B. Knowledge management enablers, processes, and organizational performance: an integrative view and empirical examination. Journal of Management Information Systems, [S.1.], v. 20, n. 1, p. 179-228, 2003.

LICHTENTHALER, U.; LICHTENTHALER, E. A capability-based framework for open innovation: complementing absorptive capacity. Journal of Management Studies, [S.1.], v. 46, n. 8, p. 35-52, 2009.

LOBIANCO, M. M. L.; RAMOS, A. S. M. Uso da internet no setor de hotelaria de recife-PE. RAE- Eletrônica, [S.1.], v. 3, n. 2, p. 16, 2004.

LUMPKIN, G. T.; DESS, G. G. Clarifying the entrepreneurial orientation construct and linking it to performance. Academy of Management Review, [S.1.], v. 21, n. 1, p. 135-172, 1996.

MARIN-GARCIA, J.; AZNAR-MAS, L.; GUEVARA, F. G. L. Innovation Types and Talent Management for Innovation. Working Papers on Operations Management, [S.1.], v. 2, n. 2, p. 25-31, 2011.

MARQUÉS, D. P.; SIMÓN, F. J. G. The effect of knowledge management practices on firm performance. Journal of Knowledge Management, [S.1.], v. 10, n. 3, p. 143-156, 2006.

MCDONALD, F. Cautions International Entrepreneurs: The case of the Mittelstand. Journal of International Entrepreneurs, [S.1.], v. 4, n. 1, p. 363-381, 2003.

MENGUC, B.; AUH, S. Development and Return on Execution of Product Innovation Capabilities: The Role of Organizational Structure. Industrial Marketing Management, [S.1.], v. 39, n. 5, p. 820-831, 2010.

MOLAS, O.; PARELLADA, M. 22@: 10 years of economic transformation. Revista Econòmica de Catalunya, Catalunya, n. 64, July, 2014.

MOLERO, J.; GARCIA, A. The innovative activity of foreign subsidiaries in the Spanish Innovation System: An evaluation of their impact from a sectoral taxonomy approach. Technovation, [S.1.], v. 28, p. 739-757, 2008. 
MONTORO-SÁNCHEZ, M. Á.; MORA-VALENTÍN, E. M.; URBINA-CRIADO, M. O. de. Localización en parques científicos y tecnológicos y cooperación en $\mathrm{I}+\mathrm{D}+\mathrm{i}$ como factores determinantes de la innovación. Revista Europea de Dirección y Economía de la Empresa, [S.I.], v. 2, p. 182-190, 2012.

MORÉ, R. P. O. et al. A capacidade absortiva e inovatividade em ambientes tecnológicos: uma análise a luz da teoria. X CONGRESO INTERNACIONAL SOBRE TECNOLOGIA, CONOCIMIENTO Y SOCIEDAD, Madrid, 6-7 febrero, 2014. Anais... Madrid, 2014.

MOUSTAGHFIR, K. The dynamics of knowledge assets and their link with firm performance. Measuring Business Excellence, [S.1.], v. 12, n. 2, p. 10-24, 2008. MOTTA, F. C. P.; VASCONCELOS, I. F. G. Teoria geral da administração. São Paulo: Pioneira Thomson Learning, 2004.

NOHRIA, N.; ECCLES, R. Networks and organizations. Boston: Harvard Business School Press, 1992.

NONAKA, I.; VON KROGH, G.; VOELPEL, S. Organizational knowledge creation theory: evolutionary paths and future advances. Organization studies, [S.1.], v. 27, n. 8, p. 1.179-1.208, 2006.

NONAKA, I.; VON KROGH, G. Tacit knowledge and knowledge conversion: controversy and advancement in organizational knowledge creation theory. Organization Science, [S.1.], v. 20, n. 3, p. 635-652, 2009.

OECD. Organisation for economic co-operation and development. Oslo Manual: guidelines for collecting and interpreting innovation data. 3. ed. Paris: OECD, 2005.

PADILLA-PÉREZ, R.; GAUDIN, Y. Science, technology and innovation policies in small and developing economies: The case of Central America. Research Policy, [S.1.], v. 43, p. 749-759, 2014.

PAVITT, K. Innovation Processes. In: FAGERBERG, J.; MOWERY, D. C.; NELSON, R. R. The Oxford

Handbook of Innovation. Oxford: Oxford University Press, 2004.

PIQUÉ, J. M. et al. Open innovation market places. a tool kit for organising open innovation market places (OIMP) events. IASP World Conference, Doha; Qatar, October 2014.
RAMAMMURTI, R.; SINGH, J. (Ed.). Emerging

Multinationals in Emerging Markets. England: Oxford University Press, 2009.

REED, R.; DEFILLIPPI, R. J. Causal ambiguity, barriers to imitation and sustainable competitive advantage.

Academy of Management Review, [S.I.], v. 15, n. 1, p. 88-102, 1990.

ROTHWELL, R. Industrial innovation: success, strategy, trends. In: DODGSON, M.; ROTHWELL, R. (Ed.) The handbook of industrial innovation. Hants: Edward Elgar Publishing, 1994.

SIRKIN, H.; HEMERLING, J.; BHATTACHARYA, A. Globality: Competing with Everyone from Everywhere for Everything. New York: Business Plus, 2008.

SPENDER, J. C. Making knowledge the basis of a dynamic theory of the firm. Strategic Management Journal, [S.1.], v. 17, p. 45-62, 1996.

SUBRAMANIAN, A. Innovativeness: redefining the concept. Journal of Engineering and Technology Management, [S.1.], v. 13, n. 3, p. 223-243, 1996.

TAJEDDINI, K.; TRUEMAN, M.; LARSEN, G. Examining the effect of market orientation on innovativeness.

Journal of Marketing Management, [S.1.], v. 22, p. 529551, 2006.

TEECE, D. J. Strategies for managing knowledge assets: the role of firm structure and industrial contex. Long Range Planning, [S.1.], v. 33, p. 65, 2000.

TIDD, J.; BESSANT, J.; PAVITT, K. Gestão da inovação. 3. ed. Porto Alegre: Bookman, 2008.

TODOROVA, G.; DURISIN, B. Absorptive capacity: Valuing a reconceptualization. Academy of Management Review, [S.1.], v. 32, p. 774-786, 2007.

VAHLNE, J.; JOHANSON, J. The Uppsala model on evolution of the multinational business enterprise: from internalization to coordination of networks. International Marketing Review, [S.1.], v. 30, n. 3, p. 189-210, 2013.

VASUDEVA, G.; SPENCER, J. W.; TEEGEN, H. J. Bringing the institutional context back in: a cross-national comparison of alliance partner selection and knowledge acquisition.

Organization Science, [S.1.], v. 24, n. 2, p. 319-338, 2013. 
YANGA, C. H.; MOTOHASHIB, K.; CHENC, J. R. Are new technology-based firms located on science parks really more innovative? Evidence from Taiwan. Research Policy, [S.1.], v. 38, p. 77-85, 2009.

WATTS, F. et al. Validation of a rubric to assess innovation competence. Working Papers on Operations

Management, [S.1.], v. 3, n. 1, p. 25-31, 2012.

WELCH, L.S.; LUOSTARINEN, R.K. Internationalization: evolution of a concept. In: BUCKLEY, P. J; GHAURI, P. $N$. (Ed). The internationalization of the firm: a reader. London: Academic Press, 1993.

\section{WIND, Y.; CROOK, C. A Força dos Modelos}

Mentais. Porto Alegre: Bookman, 2004.

WISCHNEVSKY, J. D.; DAMANPOUR, F.; MENDEZ, F. Influence of Environmental Factors and Prior Changes on the Organizational Adoption of Changes in Products and in Technological and Administrative Processes. British Journal of Management, [S.1.], v. 4, 2010.

ZACK, M.; MCKEEN, J.; SINGH, S. Knowledge management and organizational performance: an exploratory analysis. Journal of Knowledge Management, [S.1.], v. 13, n. 6, p. 392-409, 2009.

ZAHRA, A.; GEORGE, Absorptive capacity: a review, reconceptualization, and extension. Academy of

Management Review, [S.1.], v. 17, n. 2, p. 185-203, 2002. 